\title{
ON COMPACT PERTURBATIONS AND COMPACT RESOLVENTS OF NONLINEAR m-ACCRETIVE OPERATORS IN BANACH SPACES
}

\author{
ATHANASSIOS G. KARTSATOS
}

(Communicated by Palle E. T. Jorgensen)

\begin{abstract}
Several mapping results are given involving compact perturbations and compact resolvents of accretive and m-accretive operators. A simple and straightforward proof is given to an important special case of a result of Morales who has recently improved and/or extended various results by the author and Hirano. Improved versions of results of Browder and Morales are shown to be possible by studying various homotopies of compact transformations.
\end{abstract}

\section{INTRODUCTION-PRELIMINARIES}

In what follows, the symbol $X$ stands for a real Banach space with norm $\|\cdot\|$ and (normalized) duality mapping $J$. An operator $T: X \supset D(T) \rightarrow 2^{X}$ is called "accretive" if for every $x, y \in D(T)$ there exists $j \in J(x-y)$ such that

$$
\langle u-v, j\rangle \geq 0
$$

for all $u \in T x, v \in T y$. An accretive operator $T$ is "m-accretive" if $R(T+\lambda I)=X$ for all $\lambda \in(0, \infty)$. We denote by $B_{r}(0)$ the open ball of $X$ with center at zero and radius $r>0$.

One of our purposes in this paper is to prove the following theorem:

Theorem 1. Let $T: X \supset D(T) \rightarrow 2^{X}$ be m-accretive and $C: \overline{D(T)} \rightarrow X$ compact and uniformly continuous on bounded sets. Assume that there exist positive constants $b, r$ such that, given $p \in \overline{B_{r}(0)}$ and $x \in D(T)$ with $\|x\| \geq b$, there exists $j=j(p, x) \in J x$ such that

$$
\langle u+C x-p, j\rangle \geq 0
$$

for all all $u \in T x$. Then $\overline{B_{r}(0)} \subset \overline{(T+C)\left(B_{b}(0) \cap D(T)\right)}$.

This result improves, when $C$ is uniformly continuous on bounded sets, a relevant result of Hirano [6, Theorem 2], a result of the author [8, Theorem 3] and the following theorem of Morales in [13] .

Received by the editors February 22, 1992 and, in revised form, March 30, 1992.

1991 Mathematics Subject Classification. Primary 47H17; Secondary 35B20, 47B07, 47H06.

Key words and phrases. Accretive operator, m-accretive operator, compact perturbation, compact resolvent, Leray-Schauder degree theory. 
Theorem 2 (Morales [13]). Let $X$ be a Banach space, $T: D(T) \subset X \rightarrow 2^{X}$ $m$-accretive, and $C: \overline{D(T)} \rightarrow X$ compact. Suppose that there exist positive constants $b, r$ such that for every $x \in D(T)$ with $\|x\| \geq b$ there exists $j \in J x$ satisfying

$$
\langle u+C x, j\rangle \geq r\|x\|
$$

for all $u \in T x$. Then $\overline{B_{r}(0)} \subset \overline{(T+C)\left(\overline{B_{b}(0)} \cap D(T)\right)}$.

It is easy to see that condition $(* *)$ implies condition $(*)$ but with the same $j \in J X$ working for all $p \in \overline{B_{r}(0)}$. We can also show that Theorem 1 can be proved by using Theorem 2 , but our intention here is to give a simple and straightforward proof of Theorem 1. Hirano [6, Theorem 2] gave a rather involved proof of his result, and several other results of his in [6] are dependent on that proof. The same is true for Morales's results in [13] in connection with Theorem 2 above. Our proof of Theorem 1 actually involves only the verification of the fact that a certain mapping is a homotopy of compact transformations. The author showed in [8, Theorem 3] that Hirano's result [6, Theorem 2] is true, with a much simpler proof, under the additional assumption that $D(T)$ is absorbing (i.e., $x \in D(T)$ implies $\lambda x \in D(T)$ for every $\lambda \in(0,1)$ ). Although the author considered single-valued mappings in [8], his results hold equally well for multivalued operators.

For an m-accretive operator $T$, the "resolvents" $J_{\lambda}: X \rightarrow D(T)$ of $T$ are defined by $J_{\lambda}=(I+\lambda T)^{-1}$ for all $\lambda \in(0, \infty)$. The "Yosida approximants" $T_{\lambda}: X \rightarrow X$ of $T$ are defined by $T_{\lambda}=\frac{1}{\lambda}\left(I-J_{\lambda}\right)$. For $x \in X$, we define $|T x|$ by $|T x|=\lim _{\lambda \rightarrow 0}\left\|T_{\lambda} x\right\|$. Some of the main properties of $J_{\lambda}$ and $T_{\lambda}$ are:

1. $\left\|J_{\lambda} x-J_{\lambda} y\right\| \leq\|x-y\|$ for all $x, y \in X$.

2. $\left\|J_{\lambda} x-x\right\|=\lambda\left\|T_{\lambda} x\right\| \leq \lambda \inf \{\|y\| ; y \in T x\}$ for all $x \in D(T)$.

3. $T_{\lambda}$ is m-accretive on $X$ and $\left\|T_{\lambda} x-T_{\lambda} y\right\| \leq \frac{2}{\lambda}\|x-y\|$ for all $\lambda>0$, $x, y \in X$.

4. $T_{\lambda} x \in T J_{\lambda} x$ for all $x \in X$.

5. $\left\|T_{\lambda} x\right\| \leq|T x|$ for all $x \in D(T)$.

For these facts the reader is referred to Barbu [1] and Lakshmikantham and Leela [11].

In what follows, "continuous" means "strongly continuous" and the symbol " $\rightarrow$ " (" $\rightarrow$ ") means strong (weak) convergence. The symbol $R\left(R_{+}\right)$stands for the set $(-\infty, \infty)([0, \infty))$ and the symbols $\partial D, \bar{D}$ denote the strong boundary and the strong closure of the set $D$, respectively. An accretive operator $T$ is called "strongly accretive" if there exists a constant $\alpha>0$ such that for each $x, y \in D(T)$ there exists $j \in J(x-y)$ such that $\langle u-v, j\rangle \geq \alpha\|x-y\|^{2}$ for all $u \in T x, v \in T y$. An operator $T: X \supset D(T) \rightarrow X$ is "bounded" if it maps bounded subsets of $D(T)$ onto bounded sets. It is "compact" if it is continuous and maps bounded subsets of $D(T)$ onto relatively compact sets. It is called "demicontinuous" ("completely continuous") if it is strong-weak (weak-strong) continuous on $D(T)$. 
Definition 1. Let $U:[0,1] \times Q \rightarrow X$, where $Q$ is a bounded subset of $X$. We say that the mapping $U(t, x)$ is $Q$-continuous at $t_{0}$ if for every $\epsilon>0$ there exists $\delta\left(\epsilon, t_{0}\right)>0$ with the property: $\left\|U(t, x)-U\left(t_{0}, x\right)\right\|<\epsilon$ for every $t \in[0,1]$ with $\left|t-t_{0}\right|<\delta\left(\epsilon, t_{0}\right)$ and every $x \in Q$. We say that $U$ is $Q$ continuous on $[0,1]$ if for every $\epsilon>0$ there exists $\delta(\epsilon)>0$ with the property: $\left\|U\left(t_{1}, x\right)-U\left(t_{2}, x\right)\right\|<\epsilon$ for every $t_{1}, t_{2} \in[0,1]$ with $\left|t_{1}-t_{2}\right|<\delta(\epsilon)$ and every $x \in Q$.

We now state a fundamental lemma that is an easy consequence of the LeraySchauder theory. It can be found in Lloyd [12, Theorem 4.4.11].

Lemma 1. Let $X$ be a Banach space, and consider the equation

$$
u-U(t, u)=0 \text {, }
$$

for which we assume the following:

(i) $U:[0,1] \times Q \rightarrow X$ is compact in its second variable for each $t \in[0,1]$, where $Q$ is a closed, convex, and bounded subset of $X$ containing the origin in its interior. Furthermore, $U(t, x)$ is $Q$-continuous on $[0,1]$.

(ii) $U(0, \partial Q) \subset Q$.

(iii) $U(t, x) \neq x, t \in[0,1), x \in \partial Q$.

Then (1.1) has a solution in $Q$ for $t=1$.

\section{MAin Results}

Theorem 1 is just a trivial corollary of the more informative Theorem 3 below.

Theorem 3. Let $T: X \supset D(T) \rightarrow 2^{X}$ be m-accretive and $C: \overline{D(T)} \rightarrow X$ compact and uniformly continuous on bounded sets. Let $p \in X$, and assume that there exists a positive constant $b$ such that $x \in D(T)$ and $\|x\| \geq b$ imply that there exists $j \in J x$ such that

$$
\langle u+C x-p, j\rangle \geq 0
$$

for all $u \in T x$. Then $p \in \overline{(T+C)\left(B_{b}(0) \cap D(T)\right)}$.

Proof. We may (and do) assume that $0 \in T x_{0}$ for some $x_{0} \in D(T)$. Otherwise, we pick $x_{0} \in D(T)$ and consider instead the mappings $T_{1} x \equiv T x-v, C_{1} x \equiv$ $C x+v$, where $v$ is some point in $T x_{0}$. These mapping have exactly the same properties as $T, C$. We fix $p \in \overline{B_{r}(0)}$, and we consider the approximate problem

$$
T x+C x+\frac{1}{n} x \ni p
$$

for $n=1,2, \ldots$. We observe that this equation can be rewritten as

$$
u+C\left(T+\frac{1}{n} I\right)^{-1} u-p=0
$$

or

$$
u+\left(C(n T+I)^{-1}\right)(n u)-p=0 .
$$

In order to apply Lemma 1 , we consider the equation

$$
u+t\left[\left(C(\operatorname{tn} T+I)^{-1}\right)(n u)-p\right]=0
$$

with $t \in[0,1]$, and the mapping

$$
U(t, u) \equiv t\left[\left(C(\operatorname{tn} T+I)^{-1}\right)(n u)-p\right] .
$$


We define $U(0, x)=0, x \in X$, and observe that $U(t, x)$ is compact in $x$ for all $t \in[0,1]$. Let $Q$ denote any bounded subset of $X$. We show that the mapping $U(t, u)$ is $Q$-continuous on $[0,1]$. To this end, we first let $t_{0} \in$ $(0,1]$, and we show that $U(t, u)$ is $Q$-continuous at $t_{0}$. In fact, for $t \in$ $(0,1], u \in Q$, let

$$
y_{t_{0}}(u)=\left(t_{0} n T+I\right)^{-1} n u, \quad y_{t}(u)=(\operatorname{tn} T+I)^{-1} n u .
$$

Then

$$
y_{t_{0}}(u)=n u-t_{0} n T_{t_{0} n}(n u), \quad y_{t}(u)=n u-\operatorname{tn} T_{t n}(n u)
$$

and

$$
y_{t}(u)-y_{t_{0}}(u)=-n\left(t T_{t n}(n u)-t_{0} T_{t_{0} n}(n u)\right)
$$

Choosing $j \in J\left(y_{t}(u)-y_{t_{0}}(u)\right)$ properly, we obtain

$$
\begin{aligned}
\left\|y_{t}(u)-y_{t_{0}}(u)\right\|^{2}= & -n\left\langle t T_{t n}(n u)-t_{0} T_{t_{0} n}(n u), j\right\rangle \\
= & -n t\left\langle T_{t n}(n u)-T_{t_{0} n}(n u), j\right\rangle \\
& -n\left\langle t T_{t_{0} n}(n u)-t_{0} T_{t_{0} n}(n u), j\right\rangle \\
\leq & n\left|t-t_{0}\right|\left\|T_{t_{0} n}(n u)\right\|\left\|y_{t}(u)-y_{t_{0}}(u)\right\|,
\end{aligned}
$$

where we have used the fact that $T_{t n}(n u) \in T J_{t n}(n u)=T y_{t}(u), T_{t_{0} n}(n u) \in$ $T J_{t_{0} n}(n u)=T y_{t_{0}}(u)$ and the accretiveness of $T$. To estimate $\left\|T_{t_{0} n}(n u)\right\|$, we observe that

$$
\left\|T_{t_{0} n}(n u)\right\|=\left\|T_{t_{0} n}(n u)-T_{t_{0} n}\left(x_{0}\right)\right\| \leq \frac{2}{t_{0} n}\left\|n u-x_{0}\right\|
$$

because

$$
t_{0} n\left\|T_{t_{0} n} x_{0}\right\|=\left\|x_{0}-J_{t_{0} n} x_{0}\right\| \leq \inf \left\{\|y\| ; y \in T x_{0}\right\}=0 .
$$

Combining (2.4) and (2.5), we obtain

$$
\left\|y_{t}(u)-y_{t_{0}}(u)\right\| \leq \frac{2\left|t-t_{0}\right|}{t_{0}}\left\|n u-x_{0}\right\|,
$$

which shows the $Q$-continuity of the function $y_{t}(u)$ at each $t_{0}>0$.

The boundedness of the function $y_{t}(u)$ on $(0,1] \times Q$ follows from

$$
y_{t}(u)=J_{t n}(n u)=\left[J_{t n}(n u)-J_{t n} x_{0}\right]+J_{t n} x_{0}
$$

and

$$
\left\|y_{t}(u)\right\| \leq\left\|J_{t n}(n u)-J_{t n} x_{0}\right\|+\left\|J_{t n} x_{0}\right\| \leq\left\|n u-x_{0}\right\|+\left\|x_{0}\right\| .
$$

It is easy to see now that $U(t, u) \equiv t\left[C y_{t}(u)-p\right]$ is $Q$-continuous at each $t_{0} \in(0,1]$. This follows from

$$
\begin{aligned}
\left\|t C y_{t}(u)-t_{0} C y_{t_{0}}(u)\right\| & \leq\left\|t C y_{t}(u)-t C y_{t_{0}}(u)\right\|+\left\|t C y_{t_{0}}(u)-t_{0} C y_{t_{0}}(u)\right\| \\
& \leq t\left\|C y_{t}(u)-C y_{t_{0}}(u)\right\|+\left|t-t_{0}\right|\left\|C y_{t_{0}}(u)\right\| .
\end{aligned}
$$

Using the compactness of $C$, we can also show that $\|U(t, u)\|=$ $t\left\|C y_{t}(u)-p\right\| \rightarrow 0$ as $t \rightarrow 0^{+}$uniformly with respect to $u \in Q$.

Thus, the mapping $U(t, u)$ is $Q$-continuous at any point $t \in[0,1]$. Since the interval $[0,1]$ is compact, a simple covering argument shows that $U(t, u)$ is actually $Q$-continuous on $[0,1]$.

Since for any ball $Q=\overline{B_{q}(0)}$ we have $U\left(0, \partial B_{q}(0)\right)=\{0\} \subset Q$, Lemma 1 (with $U$ replaced by $-U$ ) will be applicable here for all large balls $B_{q}(0)$ 
if we show that all possible solutions of (2.3) are bounded independently of $t \in[0,1)$. Assume that this is not true. Since the only solution of $(2.3)$ for $t=0$ is $u=0$, there exists an unbounded sequence of solutions $\left\{u_{m}\right\}_{m=1}^{\infty}$ of (2.3) with $t=t_{m} \in(0,1)$. Let

$$
x_{m}=\left(t_{m} n T+I\right)^{-1}\left(n u_{m}\right)=J_{t_{m} n}\left(n u_{m}\right) \in D(T) .
$$

Then we have that $u_{m}=t_{m} v_{m}+\frac{1}{n} x_{m}$ for some $v_{m} \in T x_{m}$ and

$$
t_{m}\left(v_{m}+C x_{m}-p\right)+\frac{1}{n} x_{m}=0 \text {. }
$$

We note that if $\left\{\left\|x_{m}\right\|\right\}_{m=1}^{\infty}$ is bounded, then the compactness of $C$ and (2.6) imply that $\left\{\left\|t_{m} v_{m}+\frac{1}{n} x_{m}\right\|\right\}_{m=1}^{\infty}$ is bounded, which contradicts the unboundedness of $\left\{\left\|u_{m}\right\|\right\}_{m=1}^{\infty}$. Consequently, $\left\{\left\|x_{m}\right\|\right\}_{m=1}^{\infty}$ is unbounded and $\left\|x_{m}\right\| \geq b$ for some $m$. This implies that there exists some $j \in J\left(x_{m}\right)$ such that

$$
\left\langle v_{m}+C x_{m}-p, j\right\rangle \geq 0 .
$$

This and (2.6) yield the contradiction:

$$
\frac{1}{n}\left\|x_{m}\right\|^{2} \leq t_{m}\left\langle v_{m}+C x_{m}-p, j\right\rangle+\frac{1}{n}\left\|x_{m}\right\|^{2}=0 .
$$

Lemma 1 implies that $(2.1)$ is solvable for each $n=1,2, \ldots$ It is easy to see, as above, that all solutions of (2.1) lie in the ball $B_{b}(0)$. Thus, we have our conclusion, $p \in \overline{(T+C)\left(B_{b}(0) \cap D(T)\right)}$.

Since Morales considers in [13] only the possibility $\overline{B_{b}(0)} \subset \overline{R(T+C)}$ or $\overline{B_{b}(0)} \subset R(T+C)$, our comments on his results refer to the conditions which are implied by his results to conclude that a single point $p$ belongs to $\overline{R(T+C)}$ or $R(T+C)$. Theorem 4 below provides an improvement of Corollary 1 and the Proposition in Morales [13]. In the Proposition of [13] $T: X \rightarrow B(X)$ is continuous, where $B(X)$ is the space of closed and bounded subsets of $X$ associated with the Hausdorff metric. Also, a condition stronger than ours holds there on $\partial B_{b}(0)$. Our proof is also different because we introduce a new homotopy $U(t, x)$.

Theorem 4. Let $T: X \supset D(T) \rightarrow 2^{X}$ be m-accretive with $0 \in D(T)$ and $C: \overline{D(T)} \cap \overline{B_{b}(0)} \rightarrow X$ compact. Let $p \in X$, and assume that there exists a constant $b>0$ such that $x \in D(T) \cap \partial B_{b}(0)$ implies that there exists $j \in J x$ such that $(*)$ is satisfied for all $u \in T x$. Then $p \in \overline{(T+C)\left(D(T) \cap \overline{B_{b}(0)}\right)}$. If, moreover, $T$ is strongly accretive, then $p \in R(T+C)$.

Proof. We may (and do) assume that $0 \in T 0$; otherwise we can use the operators $T_{1}, C_{1}$ as in the proof of Theorem 3 with $x_{0}=0$. By Lemma 31 in Rothe's book [14] ("completely continuous" means "compact" in that book), we consider the compact extension of $C$ from the closed and bounded set $\overline{D(T)} \cap \overline{B_{b}(0)}$ to the whole space $X$. We denote this extension also by $C$. We now make use of equation (2.1), which we rewrite as

$$
x=\left(T+\frac{1}{n} I\right)^{-1}(-(C x-p))=(n T+I)^{-1}(-n(C x-p)) .
$$

This equation leads to the homotopy equation

$$
x-U(t, x)=0
$$


where

$$
U(t, x) \equiv(\operatorname{tn} T+I)^{-1}(-\operatorname{tn}(C x-p)), \quad(t, x) \in[0,1] \times X,
$$

and we observe that $U(0, x) \equiv 0$ and that $U(t, x)$ is compact in $x$ for all $t \in[0,1]$. Let $Q=\overline{B_{b}(0)}$. The $Q$-continuity of $U(t, x)$ at any $t_{0} \in(0,1]$ follows from

$$
\begin{aligned}
&\left\|U(t, x)-U\left(t_{0}, x\right)\right\| \\
& \leq\left\|(\operatorname{tn} T+I)^{-1}(-\operatorname{tn}(C x-p))-\left(t_{0} n T+I\right)^{-1}(-\operatorname{tn}(C x-p))\right\| \\
&+\left\|\left(t_{0} n T+I\right)^{-1}(-\operatorname{tn}(C x-p))-\left(t_{0} n T+I\right)^{-1}\left(-t_{0} n(C x-p)\right)\right\| \\
& \leq \frac{2\left|t-t_{0}\right|}{t_{0}}\|\operatorname{tn}(C x-p)\|+n\left|t-t_{0}\right|\|C x-p\|
\end{aligned}
$$

for all $x \in Q$, where we have used our estimates from the proof of Theorem 3 for $x_{0}=0$. The $Q$-continuity of $U(t, x)$ at $t=0$ follows from

$$
\|U(t, x)\| \leq t n\|C x-p\|
$$

for all $t>0$ and all $x \in Q$. As in the proof of Theorem $3, U(t, x)$ is $Q$-continuous on $[0,1]$. To apply Lemma 1 with $Q=\overline{B_{b}(0)}$, we need to show that (2.9) has no solution on $\partial B_{b}(0)$ for any $t \in[0,1)$. Obviously, this is true for $t=0$. Let $t \in(0,1)$ and $x_{t} \in \partial B_{b}(0)$ solve (2.9). Then $x_{t} \in D(T)$. Let $j \in J\left(x_{t}\right)$ be such that (2.7) holds with $x_{m}$ replaced by $x_{t}$, and $v_{m}$ by $v_{t}$. Then (2.8) holds, with $t_{m}$ replaced by $t, x_{m}$ by $x_{t}$, and $v_{m}$ by $v_{t}$, and we have a contradiction. Thus (2.1) is solvable for each $n$ with each solution $x_{n} \in D(T) \cap \overline{B_{b}(0)}$. Now, assume that $T$ is strongly accretive. Then the compactness of $C$ implies that $T x_{n_{k}}$ is convergent, for some subsequence $\left\{x_{n_{k}}\right\}$ of $\left\{x_{n}\right\}$. The strong accretiveness of $T$ implies easily that $x_{n_{k}} \rightarrow$ (some) $x_{0} \in \overline{D(T) \cap \overline{B_{b}(0)}}$. Since $C$ is continuous and $T$ is closed, we obtain that $T x_{0}+C x_{0} \ni p$. This completes the proof.

For compact resolvents of the m-accretive operator $T$ we have the following result which improves Theorem 7 of Morales [13].

Theorem 5. Let $T: D(T) \subset X \rightarrow 2^{X}$ be m-accretive with $J_{1}=(T+I)^{-1}$ compact. Let $C: \overline{D(T)} \rightarrow X$ be continuous and bounded and $p \in X$. Assume that there exists $b>0$ such that for every $x \in D(T)$ with $\|x\| \geq b$ there exists $j \in J x$ such that $(*)$ holds for all $u \in T x$. Then $p \in R(T+C)$.

Proof. We just note here that the proof follows the steps of the proof of Theorem 3 because the mapping $U(t, x)$ is still compact for all $t \in[0,1]$ and its $Q$-continuity on $[0,1]$ (for a bounded set $Q \subset X$ ) follows now from the continuity and the boundedness of the mapping $C$. Thus, $(2.1)$ is solvable with solutions $x_{n}$ lying inside $B_{b}(0)$ as before. Now, we observe that

$$
x_{n}=(T+d I)^{-1}\left(\left(d-\frac{1}{n}\right) x_{n}-C x_{n}+p\right),
$$

for a fixed $d>0$, and the compactness of $(T+d I)^{-1}$ along with the boundedness of $C$ implies that $\left\{x_{n}\right\}$ lies inside a compact set. Thus, there exist a subsequence $\left\{x_{n_{k}}\right\}$ of $\left\{x_{n}\right\}$ and a point $\bar{x} \in \overline{D(T)} \cap \overline{B_{b}(0)}$ such that $x_{n_{k}} \rightarrow \bar{x}$ as $k \rightarrow \infty$. Since $C$ is continuous, we have $C x_{n_{k}} \rightarrow C \bar{x}$ as $k \rightarrow \infty$. Since $T$ is closed, we finally obtain $\bar{x} \in D(T) \cap \overline{B_{b}(0)}$ and $T \bar{x}+C \bar{x} \ni p$. The proof is complete. 
Theorem 6. Let $T: D(T) \subset X \rightarrow 2^{X}$ be m-accretive with $0 \in D(T)$ and $C: \overline{B_{b}(0)} \rightarrow X$ compact. Let $p \in X$, and assume that for every $x \in \partial B_{b}(0)$ there exists $j \in J X$ with

$$
\langle C x+v-p, j\rangle \geq 0,
$$

where $v$ is a fixed element of T0. Then $p \in \overline{R(T+C)}$.

Proof. As in [8, Theorem 6], we look at the approximation problem

$$
\tilde{T}_{n} x+\tilde{C} x+\alpha x=p .
$$

We have made the following conventions: $\tilde{T} x \equiv T x-v, \tilde{C} x \equiv C x+v, \alpha$ is a positive constant, $\tilde{T}_{n}=n\left(I-\tilde{J}_{n}\right)$, and $\tilde{J}_{n}=\left(I+\frac{1}{n} \tilde{T}\right)^{-1}$. Let $x \in \partial B_{b}(0), j \in$ $J x$ be such that $(* *)$ holds. Then, since $\tilde{T}_{n}(0)=0$, we have

$$
\left\langle\tilde{T}_{n} x+\tilde{C} x+\alpha x-p, j\right\rangle=\left\langle\tilde{T}_{n} x+C x+v+\alpha x-p, j\right\rangle \geq 0 .
$$

Since the operator $\tilde{T}_{n}+\alpha I$ is strongly accretive and uniformly continuous on bounded subsets of $X$ and $\tilde{C}$ is compact, Theorem 13.21 of Browder [3] says that (2.10) is solvable for each $\alpha>0$ with a solution $x_{n}$ lying inside the closed ball $\overline{B_{b}(0)}$. Since $T+\alpha I$ is strongly accretive and $\tilde{C}$ is compact, we obtain, as in the proof of Theorem 8 of [13], that $x_{n_{k}} \rightarrow$ (some) $x_{0} \in \overline{B_{b}(0)}$. Since $\tilde{T}_{n_{k}} x_{n_{k}} \rightarrow-\tilde{C} x_{0}-\alpha x_{0}+p$, we have (see Barbu [1, Proposition 3.4]) $x_{0} \in D(T)$ and $-\tilde{C} x_{0}-\alpha x_{0}+p \in \tilde{T} x_{0}$. It follows that the problem

$$
T x+C x+\alpha_{n} x \ni p
$$

is solvable for a positive sequence $\left\{\alpha_{n}\right\}$ with $\alpha_{n} \rightarrow 0^{+}$and solutions $x_{n}$ lying inside $\overline{B_{b}(0)}$. The proof is finished.

Theorem 6 is an improvement of Morales' Theorem 8 in [13]. Morales considered single-valued operators there and a stronger condition on the boundary of $B_{b}(0)$.

We are now going to establish a new result involving compact perturbations of continuous and demicontinuous accretive operators. This result improves a fundamental result of Browder [3, Theorem 13.21] dealing with continuous accretive operators in spaces $X$, with $X^{*}$ uniformly convex, or uniformly continuous accretive operators in general Banach spaces.

Theorem 7. Let $G$ be a bounded open subset of $X$ with $0 \in D$, and let $C$ : $\bar{G} \rightarrow X$ be compact. Moreover, fix $p \in X$, and assume one of the following:

(i) $X^{*}$ is uniformly convex and $T: \bar{G} \rightarrow X$ is demicontinuous, accretive, and such that

$$
\langle T x+C x-p, J x\rangle \geq 0, \quad x \in \partial G .
$$

(ii) $T: \bar{G} \rightarrow X$ is continuous, accretive, and for every $x \in \partial G$ condition (*) is satisfied with $u=T x$ for all $j \in J x$. Then $p \in \overline{R(T+C)}$.

Proof. We assume (i) and use the approximating problem

$$
T x+C x+\frac{1}{n} x=p .
$$

As before, we may (and do) assume that $T 0=0$. We first remark that the operator $\tilde{T}: x \rightarrow T x+\frac{1}{n} x$ is demicontinuous and strongly accretive on $\bar{G}$. Because 
of this, $\tilde{T} G$ is open in $X$ and $\tilde{T} \bar{G}$ is closed in $X$ by the author's invariance of domain result [7, Theorem 1]. We also have $\overline{\tilde{T} G}=\tilde{T} G \cup \partial \tilde{T} G$ and $\tilde{T} \bar{G}=\tilde{T} G \cup \tilde{T}(\partial G)$. Since $\tilde{T} \bar{G}=\overline{\tilde{T}} \bar{G} \supset \bar{T} G$ and $\tilde{T}$ is injective, we obtain that $\partial \tilde{T} G \subset \tilde{T}(\partial G)$ and $\tilde{T}^{-1}$ is defined on $\partial \tilde{T} G$ and $\tilde{T}^{-1}(\partial \tilde{T} G) \subset \partial G$. Consequently, the homotopy mapping $f_{t}(x)=x+t\left(C \tilde{T}^{-1} x-p\right)$ is well defined on $\overline{\tilde{T} G}$, and the Leray-Schauder degree $d\left(f_{t}, \tilde{T} G, 0\right)$ is also well defined for all $t \in[0,1]$, provided that $0 \notin f_{t}(\partial \tilde{T} G)$, because $0 \in \tilde{T} G$ and the range of the mapping $x \rightarrow C \tilde{T}^{-1} x-p$ on $\bar{T} G$ is a relatively compact subset of $X$. The reader should note that we have not assumed that $\tilde{T} G$ is a bounded set and refer to Browder [3, p. 183] and Lloyd [12, Remarks, p. 59] for further information on this degree. To show that $(2.11)$ is solvable, it suffices to show that $f_{t}$ has no zero on $\partial \tilde{T} G$ for any $t \in[0,1)$. This is certainly true for $t=0$. Assume that $x_{t} \in \partial \tilde{T} G$, for some $t \in(0,1)$, and let $u_{t}=\left(T+\frac{1}{n} I\right)^{-1} x_{t} \in \partial G$. Then

$$
T u_{t}+t C u_{t}+\frac{1}{n} u_{t}=t p .
$$

We now show that

$$
\left\langle T u_{t}+t\left(C u_{t}-p\right), J u_{t}\right\rangle \geq 0
$$

for all $t \in(0,1)$. In fact, we have $\left\langle T u_{t}, J u_{t}\right\rangle \geq 0$. If $\left\langle C u_{t}-p, J u_{t}\right\rangle \geq 0$, our assertion is trivially true. Let $\left\langle C u_{t}-p, J u_{t}\right\rangle<0$. Then condition (A) implies

$$
\left\langle T u_{t}, J u_{t}\right\rangle \geq-\left\langle C u_{t}-p, J u_{t}\right\rangle>-t\left\langle C u_{t}-p, J u_{t}\right\rangle
$$

and $\left\langle T u_{t}+t\left(C u_{t}-p\right), J u_{t}\right\rangle>0$. Applying (2.13) to (2.12), we get the contradiction:

$$
\frac{1}{n}\left\|u_{t}\right\|^{2} \leq \frac{1}{n}\left\|u_{t}\right\|^{2}+\left\langle T u_{t}+t\left(C u_{t}-p\right), J u_{t}\right\rangle=0
$$

Thus, (2.11) is solvable with solution $u_{n}, n=1,2, \ldots$, lying in $\bar{G}$. Since $\bar{G}$ is bounded, we have $p \in \overline{R(T+C)}$.

The proof of our conclusion under (ii) is almost identical to the above in view of the invariance of domain result of Deimling [5, Theorem 3]. It is therefore omitted.

The condition that $(*)$ be satisfied "for all $j \in J x$ " in Theorem 7 can be reduced to the same condition but "for some $j \in J x$ " under one of the following additional assumptions:

(a) $\langle T x-T y, j\rangle \geq 0$ for all $x, y \in D(T), j \in J(x-y)$.

(b) $T: X \supset G_{1} \rightarrow X$ is continuous and accretive, where $G_{1} \supset \bar{G}$ is open.

Actually, (b) implies (a), by Theorem 9.4 of Browder [3], because the Cauchy problem $x^{\prime}+T x=0, x(0)=v$, is solvable for all $v \in G_{1}$ (cf. Deimling [5, proof of Theorem 3]).

\section{DisCUSSION-EXAMPLE}

We let $\Omega$ denote a bounded domain in $R^{n}$ with smooth boundary and consider the problem

$$
-\Delta \rho(u(x))+\phi\left(\|u\|_{L^{1}}\right) g(x, u(x))=p(x), \quad \text { a.e. } x \in \Omega .
$$


Example 1. Consider (P) with the following assumptions:

(i) $\rho \in C(R) \cap C^{1}(R \backslash\{0\})$ is nondecreasing and such that $\rho(0)=0$ and, for some constants $K>0, \alpha \geq 1$,

$$
\rho^{\prime}(t) \geq K|t|^{\alpha-1}, \quad t \in R \backslash\{0\} .
$$

(ii) $g: \Omega \times R \rightarrow R$ is continuous and such that

$$
|g(x, u)| \leq q(x)+q_{1}|u|,
$$

where $q: \Omega \rightarrow R_{+}$is in $L^{1}(\Omega)$ and $q_{1}$ is a positive constant.

(iii) $\phi: R_{+} \rightarrow R_{+}$is continuous.

(iv) $p \in L^{1}(\Omega)$ has the following property: there exists a constant $b>0$ such that for each $u \in L^{1}(\Omega)$ with $\|u\|_{L^{1}} \geq b$ and each $j \in J u$ we have

$$
\int_{\Omega}\left[\phi\left(\|u\|_{L^{1}}\right) g(x, u(x))-p(x)\right] j(x) d \mu(x) \geq 0 .
$$

Then $(\mathrm{P})$ has a solution $u \in L^{1}(\Omega)$ with $\rho(u) \in W_{0}^{1,1}(\Omega)$.

Proof. Consider the operator $T: L^{1}(\Omega) \supset D(T) \rightarrow L^{1}(\Omega)$ defined by $(T u)(x)$ $\equiv-\Delta \rho(u(x))$, where $D(T)=\left\{u \in L^{1}(\Omega) ; \rho(u) \in W_{0}^{1,1}(\Omega), \Delta \rho(u) \in\right.$ $\left.L^{1}(\Omega)\right\}$. Also, consider the operator $C$ defined by

$$
(C u)(x)=\phi\left(\|u\|_{L^{1}}\right) g(x, u(x)) .
$$

Bénilan has shown in [2] that $T$ is m-accretive and $\overline{D(T)}=L^{1}(\Omega)$. Vrabie has shown in [16, Lemma 2.6.2] that $T$ generates a compact semigroup on $\overline{D(T)}$, which implies that $(T+I)^{-1}$ is a compact operator on $L^{1}(\Omega)$. Vainberg's Theorem 19.1 in [15] says that the operator $C$ is continuous and bounded on all of $L^{1}(\Omega)$. Our conclusion follows from Theorem 5 for $X=L^{1}(\Omega)$.

In the above example we may take $g(x, u)=(2+\sin u) u, \phi(t)=\frac{1}{1+t}$. Then (P) is solvable as in Example 1 for all functions $p \in L^{1}(\Omega)$ with $\|p\|_{L^{1}}<1$. In fact, we first note that $J u \subset L^{\infty}(\Omega)$ is given by

$$
J u=\|u\|_{L^{1}} \operatorname{SGN}(u),
$$

where

$$
\operatorname{SGN}(u)= \begin{cases}1, & u>0 \\ {[-1,1],} & u=0 \\ -1, & u<0\end{cases}
$$

(cf. Barbu [1, p. 161]). Let $j \in J u$ with $\langle T u, j\rangle \geq 0$. Then we have

$$
\begin{aligned}
\langle T u+C u-p, j\rangle & \geq \int_{\Omega}\left[\frac{(2+\sin (u(x))) u(x)}{1+\|u\|_{L^{1}}}-p(x)\right] j(x) d \mu(x) \\
& \geq\|u\|_{L^{1}} \int_{\Omega}\left[\frac{|u(x)|}{1+\|u\|_{L^{1}}}-|p(x)|\right] d \mu(x) \\
& =\|u\|_{L^{1}}\left[\frac{\|u\|_{L^{1}}}{1+\|u\|_{L^{1}}}-\|p\|_{L^{1}}\right],
\end{aligned}
$$

where we have used the fact that $u(x) j(x)=\|u\|_{L^{1}}|u(x)|$, a.e. $x \in \Omega$, for all $j \in J u$. Since $\frac{t}{1+t} \rightarrow 1$ as $t \rightarrow \infty$, our assertion is true. Actually, here we have $p \in(T+C)\left(\overline{B_{b}(0)} \cap D(T)\right)$, where $b=\|p\|_{L^{1}} /\left(1-\|p\|_{L^{1}}\right)$. 
Naturally, a large number of examples can now be constructed of functions $g, \phi$ that satisfy the relevant assumptions of Theorem 5 . For the problem $T u+C u \ni p$ with $T=-\Delta$ or m-accretive and $C$ the realization in $L^{p}(\Omega)$ of an m-accretive function $\beta: R \rightarrow R$, the reader is referred to Proposition 3.7 and Theorem 3.3 in the book of Barbu [1].

Obviously, the conclusion that " $p \in \bar{D}$ " in the various results of this paper, where $D$ is some subset of $\overline{R(T+C)}$, can be replaced by " $p \in D$ " under various assumptions of strong accretiveness or $\phi$-expansiveness (cf. [7]) for the operator $T$ and/or complete continuity for the operator $C$. Also, assumptions might need to be made involving compact resolvents of $T$ and/or convexity properties of the spaces $X, X^{*}$. A result in this direction was given by the author in [8, Lemma 1]. For the sake of completeness, we give below an easy extension of it to multivalued operators $T$.

Lemma 2. Let $X$ be uniformly convex. Let $T: X \supset D(T) \rightarrow 2^{X}, C: \overline{D(T)} \rightarrow$ $X$ be m-accretive and completely continuous, respectively. Assume that there exists a sequence $\left\{\alpha_{n}\right\}$ of positive constants and a sequence $\left\{x_{n}\right\} \subset \overline{B_{b}(0)} \cap D(T)$ such that $\alpha_{n} \rightarrow 0$ and $x_{n} \rightarrow 0$ as $n \rightarrow \infty$ and

$$
u_{n}+C x_{n}+\alpha_{n} x_{n}=p
$$

for some sequence $u_{n} \in T x_{n}$. Then $x_{0} \in D(T)$ and $u_{n} \rightarrow u_{0}=-C x_{0}+p$, where $u_{0} \in T x_{0}$.

For a survey article on recent results on accretiveness and compactness, the reader is referred to [9]. For applications to the control of various equations with preassigned responses, we cite the paper [10].

\section{REFERENCES}

1. V. Barbu, Nonlinear semigroups and differential equations in Banach spaces, Noordhoff, Leyden, The Netherlands, 1975.

2. P. Bénilan, Équations d'évolution dans un espace de Banach quelconque et applications, Thése, Orsay, 1972.

3. F. Browder, Nonlinear operators and nonlinear equations of evolution in Banach spaces, Proc. Sympos. Pure Appl. Math., vol. 18, part 2, Amer. Math. Soc., Providence, RI, 1976.

4. B. D. Calvert and A. G. Kartsatos, On the convexity of the interior of the domain of an m-accretive operator, Nonlinear Anal. TMA 12 (1988), 727-732.

5. K. Deimling, Zeros of accretive operators, Manuscripta Math. 13 (1974), 365-374.

6. N. Hirano, Some surjectivity theorems for compact perturbations of accretive operators, Nonlinear Anal. TMA 8 (1984), 765-774.

7. A. G. Kartsatos, Zeros of demicontinuous accretive operators in reflexive Banach spaces, J. Integral Equations 8 (1985), 175-184.

8. _ On the solvability of abstract operator equations involving compact perturbations of m-accretive operators, Nonlinear Anal. TMA 11 (1987), 997-1004.

9. __ Recent results involving compact perturbations and compact resolvents of accretive operators in Banach spaces, Proc. World Congr. Nonlinear Analysts (Tampa, 1992) (to appear).

10. A. G. Kartsatos and R. D. Mabry, Controlling the space with pre-assigned responses, J. Optim. Theory Appl. 54 (1987), 517-540.

11. V. Lakshmikantham and S. Leela, Nonlinear differential equations in abstract spaces, Pergamon Press, Oxford, 1981.

12. N. G. Lloyd, Degree theory, Cambridge Univ. Press, London, 1978. 
13. C. Morales, Remarks on compact perturbations of m-accretive operators, Nonlinear Anal. TMA 16 (1991), 771-780.

14. E. H. Rothe, Introduction to various aspects of degree theory in Banach spaces, Math. Surveys Monographs, vol. 23, Amer. Math. Soc., Providence, RI, 1986.

15. M. M. Vainberg, Variational methods for the study of nonlinear operators, Holden-Day, San Francisco, CA, 1964.

16. I. I. Vrabie, Compactness methods in nonlinear evolutions, Longman Sci. Tech., London, 1986.

Department of Mathematics, University of South Florida, TAMPa, Florida 33620-5700

E-mail address: hermes@gauss .math.usf .edu 